\title{
Vascular Determinants of Epilepsy: The Rotterdam Study
}

\author{
*Xinhua Li, *†Monique M. B. Breteler, $\dagger \neq$ Martine C. de Bruyne, $§$ Harry Meinardi, \\ $" W$. Allen Hauser, and $* \dagger$ Albert Hofman
}

\begin{abstract}
*The Netherlands Institute for Health Sciences, Erasmus University Medical School; †Department of Epidemiology \& Biostatistics, Erasmus University Medical School; and $\$$ Department of Medical Informatics, Erasmus University Medical School, Rotterdam, the Netherlands; §Department of Epileptology, Catholic University of Nijmegen, Nijmegen, the Netherlands; and "G. H. Sergievsky Centre, Columbia University, New York, New York, U.S.A.
\end{abstract}

\begin{abstract}
Summary: Purpose: To investigate the relation between vascular determinants and epilepsy in an elderly population.

Methods: This is a cross-sectional, community-based, casecontrol study. The total study population was comprised of 4,944 subjects, 65 of whom had epilepsy which conformed to International League Against Epilepsy (ILAE) criteria. Vascular determinants that were evaluated included a history of stroke or myocardial infarction, peripheral vascular disease, hypertension, serum total cholesterol and left ventricular hypertrophy. Multivariate logistic regression analysis was used to calculate prevalence odds ratios (OR), adjusted for age and gender, as a measure of the strength of the associations.

Results: A history of stroke was strongly associated with lifetime epilepsy (OR 3.3; 95\% CI [Confidence Interval] 1.38.5), as well as with late-onset epilepsy (OR 3.1; 95\% CI 0.910.6). All vascular determinants were associated with lifetime
\end{abstract}

epilepsy and late-onset epilepsy, with odds ratios $>1$. When stroke patients were excluded, the odds ratios were statistically significant for the relationships between total cholesterol and late-onset epilepsy (OR 1.3, 95\% CI 1.0-1.6) and left ventricular hypertrophy and late-onset epilepsy (OR 2.9, 95\% CI 1.08.6). Furthermore, presence of any of these vascular indicators was twice as common among subjects with late-onset epilepsy as compared with subjects without epilepsy (OR $2.0,95 \% \mathrm{CI}$ $0.9-4.2$ ), and this was statistically significant when stroke patients were excluded (OR 2.1, 95\% CI 1.0-4.7).

Conclusions: These results suggest that there may be a relationship between vascular factors and the risk of late-onset epilepsy, apart from the relationship that exists through clinically overt stroke. Key Words: Epilepsy-EpidemiologyCase control study-Risk factors-Vascular factors.
Epilepsy is one of the most common neurological disorders in the elderly (1-3). From the 1960 s to the 1980 s, the incidence of epilepsy decreased among children and increased among elderly subjects $(4,5)$. Most cases of epilepsy are idiopathic or cryptogenic, and in only 40$50 \%$, the epilepsy is attributed to an identified cause $(5,6)$. In the elderly, stroke is the most commonly identified cause of epilepsy $(3,7-9)$. It has been suggested that other vascular determinants or silent infarcts may underlie some of the idiopathic or cryptogenic cases in the elderly $(10-12,23)$. We conducted a populationbased cross-sectional, case-control study to explore the relationship between vascular determinants and epilepsy in the elderly.

\footnotetext{
Accepted May 13, 1997

Address correspondence and reprint requests to Dr. M. M. B. Breteler at Department of Epidemiology \& Biostatistics, Erasmus University Medical School, PO Box 1738,3000 DR Rotterdam, The Netherlands.
}

\section{SUBJECTS AND METHODS}

\section{Study design}

The study was conducted as part of the Rotterdam Study, a community based prospective followup study of all persons of $\geqslant 55$ years, living in the district of Ommoord in Rotterdam (13). The baseline survey took place from June 1990 to June 1993. From June 1991 on, all subjects were screened for epilepsy (2). Throughout the Rotterdam Study, subjects were surveyed in random clusters. The total cohort consisted of 7,983 participants (response $78 \%$ ), and 5,559 of these were seen in the period in which we screened for epilepsy. The standard Rotterdam Study protocol was comprised of a home interview, followed by physical examinations conducted in a research center. The screening and diagnostic procedures for epilepsy have been described in detail elsewhere (2). In short, the screening for epilepsy was conducted during the home interview by a trained research assistant and consisted of 1 question, directly asking whether the subject had epilepsy or had epileptic seizures, and 3 questions about possible symptoms of epi- 
lepsy, that were taken from the World Health Organization (WHO) research protocol for screening of neurological disorders in developing countries (14). In addition, all current drug prescriptions were registered and classified according to the Anatomical Therapeutic Chemical (ATC) classification index (15). Screenpositives were persons who themselves reported epilepsy or seizures, or used antiepileptic drugs (ATC code N03), or answered "yes" to at least 2 of the 3 WHO questions.

For all screen-positives, additional information was obtained from medical records of general practitioners and specialists including EEG and CT scan reports, orif no further information were available from medical records - through a telephone interview conducted by a specially trained physician who took a detailed medical and seizure history. If indicated, the subject was reinterviewed by 1 of 4 epileptologists. All 313 screen-positives were subsequently reviewed by a panel consisting of the study physician and the 4 epileptologists, which decided whether a subject had epilepsy and, if so, classified the epilepsy according to the classification of the ILAE (16). For the current study, we included as cases subjects who were identified with a diagnosis of epilepsy according to ILAE criteria (16). The reference population consisted of all screen-negative subjects $(n=5246)$ and of the screen-positive subjects in whom a diagnosis of epilepsy could be excluded $(n=220)$. Patients with single acute symptomatic seizures were excluded from the study (n $=7$ ). We also excluded all subjects (patients and controls) with dementia, because information about the various risk factors could not be obtained reliably, in most instances. Furthermore, we may have misclassified some demented epilepsy cases as nonepileptic, due to underreporting of seizures and symptoms. This left 65 cases and 4,944 reference subjects.

Of the cases, 27 had localization-related epilepsy, 5 had generalized epilepsy, 20 had undetermined epilepsy, and 13 were classified as special syndromes because they had suffered from a cryptogenic seizure, after which antiepileptic drug (AED) treatment was started.

\section{Measurements}

The baseline examinations of the Rotterdam Study included an interview at each participant's home, during which information on current health status, medical history, drug prescriptions and actual use, smoking behavior, and other information, was obtained by means of a computerized questionnaire; this was followed in each case by several clinical examinations at a research center.

A history of stroke, myocardial infarction, head trauma with loss of consciousness, and bacterial meningitis were assessed by direct questioning. For a diagnosis of stroke, symptoms should have been present for at least $24 \mathrm{~h}$; and confirmation of the diagnosis by a treating physician was required. Myocardial infarction was de- fined as self-reported myocardial infarction with hospital admission for myocardial infarction or electrocardiogram (ECG) confirmation. Ankle systolic blood pressure was determined with the subject in supine position at both right and left posterior tibial arteries with a Doppler ultrasound transducer, by using a random zero sphygmomanometer (cuff size $38 \times 14 \mathrm{~cm}$ ). Peripheral arterial disease was considered present when the left or right ankle to brachial systolic blood pressure ratio (anklebrachial index) was $<0.90$ (17). Serum total cholesterol was determined with an automated enzymatic procedure (18). The presence of left ventricular hypertrophy was assessed in resting 12-lead ECGs, which were made at the research center with an ACTA Gnosis IV machine (Esaote/Biomedica, Florence, Italy) and digitally stored. ECGs were analyzed by the Modular ECG Analysis System (MEANS) computer program, which has been evaluated extensively by the developers themselves and by others $(19,20)$.

\section{Analysis}

We used logistic regression analysis to calculate odds ratios as a measure of the strength of the association between each presumed risk factor and epilepsy, adjusted for age and gender. We evaluated the risk of head trauma with loss of consciousness and bacterial meningitis, to compare with the vascular determinants and especially with stroke, and as a validity check on our data. The age at onset of epilepsy was compared with the age at first occurrence of stroke, myocardial infarction, head trauma, and meningitis, to ensure that the onset of epilepsy occurred after the development of these risk factors in our study. For epilepsy patients, reported dates were checked with medical records. Subjects who developed these conditions after onset of epilepsy were classified as negative for the specific risk factor. We assessed whether vascular determinants were associated with epilepsy, independently from the relation between stroke and epilepsy, through a multivariate model in which we adjusted for stroke, as well as through an analysis from which stroke patients were excluded. We performed separate analyses for lifetime epilepsy (epilepsy at some time during life, no matter what the age at onset) and late-onset epilepsy (i.e., onset age $>40$ years).

Some studies reported that AEDs may increase the cholesterol level $(21,22)$. Therefore, we did a subanalysis to study the relationship between total cholesterol and epilepsy, for which we excluded all persons who were using AEDs at the time of the examination $(n=42)$.

Left ventricular hypertrophy results mainly from longstanding hypertension. Hesdorffer et al. reported an association between left ventricular hypertrophy and the risk of unprovoked seizures in subjects not treated with diuretics, but not in subjects treated with diuretics (23). To evaluate this in our data, we performed an analysis in 
which we adjusted additionally for antihypertensive treatment. Also, we did a subanalysis stratified according to antihypertensive treatment.

\section{RESULTS}

Table 1 shows the distribution of the study population according to age, gender, and the determinants of interest. The control group was slightly older than the case group $(\mathrm{p}=0.015)$. The proportion of women did not differ significantly between groups $(\mathrm{p}=0.45)$.

Table 2 shows the ORs and 95\% CIs for the association of each presumed risk factor with lifetime epilepsy $(\mathrm{n}=65)$ and with late-onset epilepsy $(\mathrm{n}=39)$. A history of stroke showed a statistically significant association with lifetime epilepsy, with an adjusted OR of 3.3 (95\% CI: 1.3-8.5); this changed little when confined to late--onset epilepsy. The adjusted $\mathrm{OR}$ per $\mathrm{mmol} / \mathrm{L}$ increase of serum total cholesterol was 1.1 (95\% CI: 0.91.3) for lifetime epilepsy, and 1.3 (95\% CI: 1.0-1.6) for late-onset epilepsy. After excluding the AED users, this OR became 1.0 (95\% CI: 0.8-1.3) for lifetime epilepsy, but remained 1.3 (95\% CI: 1.0-1.8) for late-onset epilepsy. When adjusted only for age and gender, the ORs of left ventricular hypertrophy were 1.5 (95\% CI: $0.5-$ 4.4) for lifetime epilepsy and 2.6 (95\% CI: 0.9-7.6) for late-onset epilepsy. These remained almost the same when we added antihypertensive drug use to the regression models, or when we stratified for antihypertensive drug use. Myocardial infarction and peripheral arterial disease showed positive associations with both lifetime epilepsy and late onset epilepsy, but these were not statistically significant. Presence of any vascular determinant, other than stroke, was twice as common among subjects with epilepsy as compared to those without. History of head trauma with loss of consciousness and bacterial meningitis were significantly associated with lifetime epilepsy, but the ORs were slightly smaller and not statistically significant when the association was confined to late-onset epilepsy. When the relationship be-

TABLE 1. Description of the study population [values are means $(S D)$ or frequency]

\begin{tabular}{lcc}
\hline \multicolumn{1}{c}{ Variates } & $\begin{array}{c}\text { Reference } \\
(\mathrm{n}=4879)\end{array}$ & $\begin{array}{c}\text { Cases } \\
(\mathrm{n}=65)\end{array}$ \\
\hline Age (yrs) & $68.5(8.8)$ & $67.6(7.8)$ \\
Female (\%) & 58.5 & 63.1 \\
Vascular determinants & & \\
History of stroke (\%) & 3.1 & 8.1 \\
Peripheral arterial disease (\%) & 17.5 & 22.8 \\
History of myocardial infarction $(\%)$ & 6.8 & 7.9 \\
Total cholesterol (mmol/l) & $6.6(1.2)$ & $6.8(1.1)$ \\
$\quad$ Left ventricular hypertrophy (\%) & 4.6 & 6.3 \\
Other risk factors & & \\
$\quad$ History of head trauma (\%) & 12.9 & 28.1 \\
History of meningitis (\%) & 0.8 & 3.2 \\
\hline
\end{tabular}

SD, Standard deviation.
TABLE 2. Odds ratios (OR) and $95 \%$ confidence intervals (95\% CI) for the association between each putative risk factor and epilepsy, adjusted for age and gender

\begin{tabular}{|c|c|c|c|c|}
\hline \multirow{2}{*}{$\begin{array}{c}\text { Epilepsy } \\
\text { covariates }\end{array}$} & \multicolumn{2}{|c|}{$\begin{array}{l}\text { Lifetime epilepsy } \\
\qquad(\mathrm{n}=65)\end{array}$} & \multicolumn{2}{|c|}{$\begin{array}{l}\text { Late-onset } \\
(\mathrm{n}=39)\end{array}$} \\
\hline & OR & $95 \% \mathrm{CI}$ & OR & $95 \% \mathrm{CI}$ \\
\hline \multicolumn{5}{|l|}{ Vascular determinants } \\
\hline Stroke & 3.3 & $1.3-8.5$ & 3.1 & $0.9-10.6$ \\
\hline Total cholesterol $(\mathrm{mmol} / \mathrm{l})$ & 1.1 & $0.9-1.3$ & 1.3 & $1.0-1.6$ \\
\hline Left ventricular hypertrophy & 1.5 & $0.5-4.4$ & 2.6 & $0.9-7.6$ \\
\hline Myocardial infarction & 1.4 & $0.5-3.5$ & 1.7 & $0.6-4.8$ \\
\hline Peripheral arterial disease & 1.6 & $0.8-3.0$ & 1.5 & $0.7-3.6$ \\
\hline Any vascular determinant ${ }^{a}$ & 1.8 & $1.0-3.2$ & 2.0 & $0.9-4.2$ \\
\hline \multicolumn{5}{|l|}{ Other factors } \\
\hline Head trauma & 2.7 & $1.6-4.7$ & 1.8 & $0.8-4.0$ \\
\hline Meningitis & 4.6 & $1.1-19.7$ & 3.6 & $0.5-27.1$ \\
\hline
\end{tabular}

${ }^{a}$ Defined as total cholesterol $>6.5 \mathrm{mmol} / \mathrm{l}$ or previous myocardial infarction or peripheral arterial disease or left ventricular hypertrophy. $\mathrm{OR}$, odds ratio; $\mathrm{Cl}$, confidence interval.

tween each vascular determinant and lifetime and lateonset epilepsy was assessed while adjusting for age, gender, and all other vascular factors, the strength of the associations remained largely the same.

To assess the relationship between vascular determinants and epilepsy independently from the relationship between stroke and epilepsy, we then excluded stroke patients from our analysis (Table 3). For most of the variables, this hardly changed the results. However, for left ventricular hypertrophy, the association became stronger, and for late-onset epilepsy statistically significant (OR 2.9; 95\% CI 1.0-8.6). The presence of any vascular determinant was significantly associated with both lifetime epilepsy (OR 1.8; 95\% CI: 1.0-3.2) and late-onset epilepsy (OR 2.1; 95\% CI: 1.0-4.7).

\section{DISCUSSION}

In this study, we assessed vascular correlates of epilepsy in an elderly population. We found, as expected, that a previous stroke was strongly associated with the risk of epilepsy, but that other vascular risk factors were also more frequent in subjects with epilepsy, and the

TABLE 3. Odds ratios (OR) and $95 \%$ confidence intervals (95\% CI) for the association between each vascular risk factor and epilepsy, adjusted for age and gender. Subjects with a previous stroke are excluded

\begin{tabular}{|c|c|c|c|c|}
\hline \multirow[b]{2}{*}{ Epilepsy covariates } & \multicolumn{2}{|c|}{ Lifetime epilepsy } & \multicolumn{2}{|c|}{ Late-onset } \\
\hline & OR & $95 \% \mathrm{CI}$ & OR & $95 \% \mathrm{CI}$ \\
\hline Total cholesterol (mmol/l) & 1.1 & $0.9-1.3$ & 1.3 & $1.0-1.6$ \\
\hline Left ventricular hypertrophy & 1.7 & $0.6-4.8$ & 2.9 & $1.0-8.6$ \\
\hline Myocardial infarction & 1.5 & $0.6-3.9$ & 1.8 & $0.6-5.4$ \\
\hline Peripheral arterial disease & 1.6 & $0.8-3.2$ & 1.5 & $0.6-3.6$ \\
\hline Any vascular determinant ${ }^{a}$ & 1.8 & $1.0-3.2$ & 2.1 & $1.0-4.7$ \\
\hline
\end{tabular}

${ }^{a}$ Defined as total cholesterol $>6.5 \mathrm{mmol} / \mathrm{l}$ or previous myocardial infarction or peripheral arterial disease or left ventricular hypertrophy. $\mathrm{CI}$, confidence interval; OR, odds ratio. 
difference was statistically significant, even in the absence of stroke.

The effect was stronger when the analyses were confined to late-onset cases, suggesting that vascular risk factors are of particular relevance for epilepsy that develops later in life. Cross-sectional case-control studies are often hampered by the potential for bias. The strength of our study is that it was population-based, and that both cases and controls underwent a careful screening for seizures and epilepsy, thereby reducing the possibility of selection-bias. Also, the likelihood of serious information bias was minimized because all vascular and other determinants were assessed as part of the baseline protocol of the Rotterdam Study, without the interviewer knowing whether the subject had epilepsy. Potentially more serious concerns are that the study is based on prevalent cases and that risk factors were assessed after onset of epilepsy. Because prevalence is the result of incidence of, as well as survival with, the disease, in an analysis based on prevalent cases, factors influencing survival may lead to spurious associations. We consider it unlikely, however, that vascular risk factors have affected survival differently in subjects with, as compared to subjects without, epilepsy. As for the assessment of risk factors, for stroke, myocardial infarction, head trauma, and meningitis, we took care to include only events that had occurred before onset of epilepsy. For some of the other vascular factors, like left ventricular hypertrophy and peripheral arterial disease, we cannot exclude that these developed partly after onset of the epilepsy. However, because it is very unlikely that these developed as a result of the epilepsy, they can be interpreted as reflecting the proneness to vascular disease.

Another possible problem is that population-based studies of the lifetime prevalence of epilepsy may suffer from recall bias and under-reporting, in particular in under-reporting of early-onset epilepsy in remission (2), and one might wonder whether this has invalidated our study. For other, well-known, risk factors for epilepsy we found ORs with a magnitude very similar to those reported by other authors. Head trauma with loss of consciousness was, in our study, associated with lifetime epilepsy with an OR of 2.7 (95\% CI 1.6-4.7) and bacterial meningitis with an OR of 4.6 (95\% CI 1.1-9.7). This fits well with results reported by Hauser, who found ORs of 2.2 and 5.0, respectively (10), and lends further credence to our data.

Stroke is commonly accepted as an important cause of seizures in the elderly $(9,24-28)$. We found some indication for a relationship with vascular determinants apart from the relationship with stroke. There are two possible explanations. First, it may be that several subjects with epilepsy and vascular determinants but without selfreported stroke, actually did have a stroke that was never recognized (silent infarct) or not reported. An alternative explanation would be that diffuse cerebral damage associated with vascular disease can make the brain more vulnerable and increase the risk of epilepsy, even without clear infarction of brain tissue. Since we did not perform brain-imaging on all our patients, we are unable to distinguish between the 2 possibilities.

In conclusion, this study suggests that vascular risk factors are associated with an increased risk of epilepsy, either through silent infarcts or through noninfarct mechanisms. This corroborates the hypothesis that vascular disease may be underlying a sizeable proportion of cryptogenic cases of epilepsy in the elderly. These findings need to be confirmed in a prospective study based on incident cases.

Acknowledgment: These investigations were supported in part by the Committee on Epilepsy of the Division for Health Research of the National Epilepsy Fund, "The power of the Tiny" (project number CLEO A-106), the NESTOR Programme for Geriatric research (Ministry of Health and Ministry of Education), the Netherlands Organisation for Scientific Research (NWO), and the Municipality of Rotterdam.

\section{REFERENCES}

1. Hauser WA, Hesdorffer DC. Epilepsy: Frequency, Causes and Consequences. New York, NY: Demos Press, 1990.

2. De la Court A, Breteler MMB, Meinardi H, Hauser WA, Hofman A. Prevalence of epilepsy in the elderly. Epilepsia 1996;37;141-7.

3. Loiseau J, Loiseau P, Duché B, Guyot M, Dartigues JF, Aublet B A survey of epileptic disorders in southwest France: Seizures in elderly patients. Ann Neurol 1990;27:232-7.

4. Tallis R, Hall G, Craig I, Dean A. How common are epileptic seizures in old age? Age Ageing 1991;20:442-8.

5. Hauser WA, Annegers JF, Kurland LT. Incidence of epilepsy and unprovoked seizures in Rochester, Minnesota: 1935-1984. Epilepsia 1993;34:453-68.

6. Forsgren L. Prospective incidence study and clinical characterization of seizures in newly referred adults. Epilepsia 1990;31:292301.

7. Forsgren L. Prevalence of epilepsy in adults in northern Sweden. Epilepsia 1992;33(3):450-8.

8. Luhdorf K, Jensen LK, Plesner AM. Etiology of seizures in the elderly. Epilepsia 1986;27:458-62.

9. Sung CY, Chu NS. Epileptic seizures in the elderly people: Aetiology and seizure types. Age Ageing 1990;19:25-30.

10. Hauser WA. Epidemiology of epilepsy. In: Gorelick PB, Alter M, ed. Handbook of Neuroepidemiology. New York, Basel, Hongkong: Marcel Dekker, Inc., 1994;315-55.

11. Roberts RC, Shorvon SD, Cox TCS, Gilliatt RW. Clinically unsuspected cerebral infarction revealed by computed tomography scanning in late onset epilepsy. Epilepsia 1988;29:190-4.

12. Ng SKC, Hauser WA, Brust JCM, Susser M. Hypertension and the risk of new-onset unprovoked seizures. Neurology 1993;43:425-8.

13. Hofman A, Grobbee DE, DeJong PTVM, Vandenouweland FA. Determinants of disease and disability in the elderly: The Rotterdam Elderly Study. Eur J Epidemiol 1991;7:403-22.

14. World Health Organization. Research protocol for measuring the prevalence of neurological disorders in developing countries. Geneva: World Health Organization, Neurosciences Program, 1981.

15. Anatomical Therapeutic Chemical (ATC) classification index. WHO Collaborating Centre for Drug Statistics Methodology 1992, Oslo.

16. Commission on classification and terminology of the International 
League Against Epilepsy: Proposal for revised classification of epilepsies and epileptic syndromes. Epilepsia 1989;30:389-99.

17. Fowkes FGR, Houseley E, Cawood EHH, Macintyre CCA, Rucklsy CV, Precott RJ. Edinburgh artery study: Prevalence of asymptomatic and symptomatic peripheral arterial disease in the general population. Int $J$ Epidemiol 1991;20:384-92.

18. Vangent CM, Vandervoort HA, De Bruyn AM, Klein F. Cholesterol determinations: A comparative study of methods with special reference to enzymatic procedures. Clin Chim Acta 1977;75: 243-6.

19. Van Bemmel JH, Kors JA, van Herpen G. Methodology of the modular ECG analysis system MEANS. Methods Inf Med 1990; 29:346-53.

20. Willems JL, Abreu LC, Arnaud P, et al. The diagnostic performance of computer programs for the interpretation of electrocardiograms. N Engl J Med 1991;325:1767-73.

21. Luoma PV, Reunanen MI, Sotaniemi EA. Changes in serum triglyceride and cholesterol levels during long-term phenytoin treatment for epilepsy. Acta Med Scand 1979;206:229-31.
22. Isojarvi JIT, Pakarinen AI, Myllyla VV. Serum lipid levels during carbamazepine medication: A prospective study. Arch Neurol 1993;50:590-3.

23. Hesdorffer DC, Hauser WA, Annegers JF, Rocca WA. Severe, uncontrolled hypertension and adult-onset seizures: A case-control study in Rochester, Minnesota. Epilepsia 1996;37:736-41.

24. Shinton RA, Gill JS, Zezulka AV, Beevers DG. The frequency of epilepsy preceding stroke: Case control study in 230 patients. Lancet $1987 ; 1: 11-2$.

25. Forsgren L, Nyström L. An incident case-referent study of epileptic seizures in adults. Epilepsy Res. 1990;6:66-81.

26. Olsen TS, Hogenhaven H, Thage O. Epilepsy after stroke. Neurology 1987;37:1209-11.

27. Ryglewicz D, Baranska-Gieruszczak M, Niedzielska K, KrystWidzgowska T. EEG and CT findings in poststroke epilepsy. Acta Neurol Scand 1990;81:488-90.

28. Shapiro IM, Neufeld MY, Korczyn AD. Seizures of unknown origin after the age of 50: Vascular risk factors. Acta Neurol Scand $1990 ; 82: 78-81$. 